\title{
Prone position increases the risk of pancreatic fistula after laparoscopic radical gastrectomy combined with heated intraperitoneal chemotherapy: a case report
}

\section{Xing An}

Hospital of Chengdu University of Traditional Chinese Medicine

\section{Xiangwen Weng}

Hospital of Chengdu University of Traditional Chinese Medicine

\section{Li Li}

Hospital of Chengdu University of Traditional CHinese Medicine

\section{Qingsong Huang}

Hospital of Chengdu University of Traditional Chinese Medicine

\section{Kunlan Long}

Hospital of Chengdu University of Traditional Chinese Medicine

\section{Shikun Deng}

Hospital of Chengdu University of Traditional Chinese Medicine

\section{Sha Wang}

Hospital of Chengdu University of Traditional Chinese Medicine

\section{Lijia Zhi ( 10400387@qq.com )}

Hospital of Chengdu University of Traditional Chinese Medicine https://orcid.org/0000-0003-20867093

\section{Case report}

Keywords: ARDS, prone position, pancreatic fistula, laparoscopic radical gastrectomy, heated intraperitoneal chemotherapy

Posted Date: October 8th, 2020

DOl: https://doi.org/10.21203/rs.3.rs-88873/v1

License: (c) (i) This work is licensed under a Creative Commons Attribution 4.0 International License. Read Full License 


\section{Abstract}

Background: There has not been reported that prone position increases the risk of postoperative pancreatic fistula. We present a case of prone position leading to hyperthermia and pancreatic fistula in a patient with acute respiratory distress syndrome(ARDS) after laparoscopic radical gastrectomy(LRG) combined with heated intraperitoneal chemotherapy(HIPEC).

Case presentation: A 68-year-old male developed moderate ARDS after LRG combined with HIPEC. Since low tidal volume and high positive end expiratory pressure(PEEP) ventilation could not improve oxygenation, prone ventilation was selected to improve heterogeneous lung injury. However, chills and fever appeared after the position change. Abdominal computed tomography (CT) showed that the mesenteric fat space in the middle abdomen was fuzzy, local exudation was increased, and the boundary of pancreas was not clear. The increase of amylase in peritoneal drainage fluid was 10 times higher than that in serum amylase. After communicating with the general surgeon, we learned that during the operation, the surgeon had opened the pancreatic capsule to clean the local lymph nodes. It was considered that prone position lead to the sharp increase of abdominal pressure, especially the change of peripancreatic pressure. The visceral organs of the abdominal cavity squeezed each other, the pancreatic tissue was compressed, the pancreatic juice extravasation occurred, and even aggravated the pancreatic fistula. In the follow-up treatment process, the patient were given continuous abdominal drainage and avoided prone position as far as possible. Since then, the patient's temperature tended to be stable. On the 10th day after the operation, the patient successfully withdrew from the ventilator and transferred to the general ward for further specialized treatment.

Conclusion: Our case adds further concerns in ARDS patients after LRG combined with HIPEC, including the monitoring of postoperative pancreatic fistula and how to perform prone ventilation more safely.

\section{Background}

Laparoscopic radical gastrectomy(LRG) combined with heated intraperitoneal chemotherapy(HIPEC) is an effective treatment for advanced gastric cancer. Pancreatic fistula and acute respiratory distress syndrome (ARDS) are common but serious complications. Prone ventilation has been used to improve oxygenation in patients with ARDS for a long time. It is crucial to determine whether prone position is associated or not with more pancreatic fistula in patients with ARDS after abdominal surgery. In this paper, we present the case of prone position leading to hyperthermia and pancreatic fistula in a patient with ARDS after LRG combined with HIPEC.

\section{Case Presentation}

A 68-year-old male presented to the digestive clinic with symptoms of fatigue for 6 months. He had no other symptoms before. The important past medical history were hypertension and coronary heart disease. After examination, he had anemia appearance, vital signs were stable, other general physical 
examination found no abnormality. Nerve, heart, lung and abdomen were normal. The BMI was $23.9 \mathrm{~kg} / \mathrm{m}^{2}$. The chest and abdominal computed tomography (CT) showed no obvious abnormality. (Fig. 1A,1D) Hemoglobin was $50 \mathrm{~g} / \mathrm{L}$ and occult blood in stool was positive. Gastroscopy showed new organisms of ulcer in gastric horn and body, and pathological biopsy showed signet ring cell carcinoma. After proper communication and informed consent, LRG and HIPEC $(0.9 \%$ normal saline $3500 \mathrm{ml}+5$ fluorouracil $1250 \mathrm{mg}$, the input temperature was set to $43^{\circ} \mathrm{C}$ ) were performed under general anesthesia. After 30 minutes of HIPEC, the peritoneal drainage fluid turned red. Considering the risk of bleeding, the hyperthermic perfusion therapy was terminated. The operation lasted for 5.5 hours, and the intraoperative blood loss was $100 \mathrm{ml}$. He had dyspnea, tachycardia(110-120 beats/min), high blood pressure $(160 / 90 \mathrm{mmHg})$ and low oxygen saturation(88\%) after extubation of endotracheal intubation. The lung was scattered with moist rales, and the oxygenation index (OI) was $158.55 \mathrm{mmHg}$.

The chest CT was indicated patchy blurry shadows in both lungs and a small amount of bilateral pleural effusion (Fig. 1B). According to 2011 Berlin ARDS diagnostic standard, he was diagnosed as moderate ARDS and then transferred to ICU for further intensive care.

In the first two days after operation, he suffered from recurrent low fever and the body temperature fluctuated from $36.5-38.1^{\circ} \mathrm{C}$. Ceftazidime, famotidine and tranexamic acid were used. He were given noninvasive ventilator and invasive mechanical ventilation successively for protective lung ventilation strategy (low tidal volume, high frequency, high positive end expiratory pressure(PEEP)). However, the improvement of oxygenation was poor, and the lowest oxygenation index was $118.97 \mathrm{mmHg}$.

On the 3rd day after the operation, the patient was given prone position ventilation treatment. After 3 hours, the patient suddenly had chills, high fever, body temperature of $39.8{ }^{\circ} \mathrm{C}$, shortness of breath and decrease of blood oxygen saturation. The prone position was immediately suspended. The increase of interleukin-6, procalcitonin and C-reactive protein, and the decrease of lymphocytes were detected. Lung or abdominal infection was considered to be the main cause. Considering the history of malignant tumor, low immunity and high risk of drug-resistant bacteria infection, the antibiotic treatment was adjusted to imipenem cilastatin $(1 \mathrm{~g} \mathrm{q} 8 \mathrm{~h})$.After that, the body temperature gradually decreased, and the oxygenation index increased to $422.14 \mathrm{mmHg}$. Because of the obvious improvement of oxygenation after prone position, the patient was given prone position ventilation again for $12.5 \mathrm{~h}$ at night. During this period, the patient's temperature fluctuated between $37.5-38.8^{\circ} \mathrm{C}$, without chills.

On the 4th day after operation, the body temperature fluctuated from $36.8-37.6{ }^{\circ} \mathrm{C}$. The Ols were maintained above $200 \mathrm{mmHg}$, so the therapeutic schedule was not adjusted.

On the 5th day after operation, the patient was given prone position ventilation again because of the decrease of oxygenation index $(110 \mathrm{mmHg})$. Two hours later, the prone position was suspended due to chills and high fever, and the body temperature rose to $39.2{ }^{\circ} \mathrm{C}$. At the same time, the patient presented mild abdominal muscle tension, upper abdominal tenderness, and the abdominal drainage volume increased significantly. There was no significant change in infection indicators. In order to exclude the 
occurrence of anastomotic leakage, the patient was given nasal feeding with a small amount of iodixanol, and chest and abdominal nonenhanced CT were performed(Fig. 1C,1E). Bilateral pleural effusion increased. Pulmonary infection remained substantially unchanged. CT image showed a massive leak of contrast agent to the esophagus, residual stomach and part of left small intestine. The pancreas was unclear contour. Under the electronic bronchoscope, white and viscous secretions were found in the dorsal segment of the left lower lobe, and a large number of watery secretions were found in bilateral bronchial lumens. Maltophilia oligomonas was cultured in bronchoalveolar lavage fluid. According to the results of drug sensitivity, minocycline $(0.1 \mathrm{~g}, \mathrm{q} 12 \mathrm{~h})$ was added for treatment. Amylase in peritoneal drainage fluid was $1218 \mathrm{U} / \mathrm{L}$ (reference range: 0-300 U/L), serum amylase was 118.7 U/L (reference range: $35-135 \mathrm{U} / \mathrm{L}$ ). Rivalta test was weakly positive. Lactate dehydrogenase in peritoneal drainage fluid was $1103.9 \mathrm{U} / \mathrm{L}$ (reference range: 0-200 U/L). After communicating with the general surgeon, we learned that during the operation, the surgeon had opened the pancreatic capsule to clean the local lymph nodes. Therefore, we considered that the cause of high fever was that the pressure of abdominal cavity increases rapidly after prone position, which lead to the occurrence of pancreatic fistula.

Therefore, in the follow-up treatment process, the patient were given continuous abdominal drainage and avoided prone position as far as possible. Since then, the patient's temperature tended to be stable. On the 10th day after the operation, the patient successfully withdrew from the ventilator and transferred to the general ward for further specialized treatment.

\section{Discussion}

ARDS is one of common pulmonary complications after radical gastrectomy. Prone position has been the most common therapeutic strategy to improve oxygenation in ARDS patients for a long time. Prone position demonstrated a strong survival benefit in patients with oxygenation index less than 150 $\mathrm{mmHg}[1]$. Consistent with previous studies, oxygenation improved significantly after prone position in this case. Unfortunately, pancreatic fistula occurred. The first retrospective multicenter study evaluating the safety and efficacy of prone position in patients with severe ARDS after abdominal surgery, found that early postoperative prone position was not associated with increased local or surgical complications, and oxygenation improved significantly after one session of prone position[2]. However, the complications were limited to repercussions on scars, draining systems and stoma, not pancreatic fistula. Therefore, whether prone position in ARDS patients after total gastrectomy increases the risk of pancreatic fistula remains to be further studied.

In 2016, the International Study Group redefined postoperative pancreatic fistula as drainage fluid amylase concentration more than three times serum amylase concentration, and redefined the classification. The "Grade A" pancreatic fistula was redefined as biochemical leakage, and only amylase in peritoneal drainage fluid increased. "Grade B" was defined as elevated pancreatic enzyme in drainage fluid and clinical symptoms (fever, abdominal pain), which have an impact on the prognosis path, such as delayed indwelling of catheter, re placement of drainage tube, need for percutaneous puncture or endoscopic treatment of peritoneal effusion associated with pancreatic fistula, and need for angiography 
or embolization for pancreatic fistula related abdominal bleeding. Pancreatic fistula was defined as "Grade C" because of organ failure, reoperation and death[3].

Pancreatic fistula is one of the most serious and potential complications after gastrectomy (4\%-6\%)[47]. A national prospective cohort study in Japan has shown that the incidence of pancreatic fistula after laparoscopic gastrectomy is higher than that of open surgery, which is associated with pressure and heat injury to the pancreas[8]. They developed a new laparoscopic method to avoid direct compression of the pancreas during suprapancreatic lymphadenectomy. During this process, the assistant pulls the connective tissue from the lower edge of the pancreas to the tail or back, instead of compressing the pancreas with gauze or sponge. This retrospective study confirmed that avoiding pancreatic pressure is one of the important methods to prevent pancreatic fistula in laparoscopic distal gastrectomy[9]. Laparoscopic assisted surgery, combined organ resection, the use of LigaSure + ultrasonic scalpel and the number of lymph node dissection were independent influencing factors of grade B pancreatic fistula after radical gastrectomy for gastric cancer[10].

In this case, the expansion of the scope of lymph node dissection and pancreatic operation during laparoscopic surgery may lead to direct pancreatic injury. In addition, thermal injury during operation, including HIPEC, is also an important cause of pancreatic fistula. HIPEC, a new adjuvant therapy, can kill tumor cells, inhibit DNA synthesis and destroy the metabolic growth of tumor cells by using the synergistic effect of hyperthermic cell thermal damage and chemotherapy drugs, effectively inhibit the growth of tumor cells after chemotherapy, and significantly reduce the local recurrence and distant metastasis rate of tumor patients. However, the high temperature of abdominal organs and the retention of chemotherapeutic drugs in the sunken part of abdominal cavity are high risk factors for systemic inflammatory response and ARDS.

The patient developed ARDS after the surgery. In the course of two times of prone position treatment, the patients had chills and high fever. It was considered that prone position leads to the increase of abdominal pressure, the mutual extrusion of abdominal organs and even aggravation of pancreatic fistula.

Amylase concentration in drainage fluid after gastric cancer surgery can be considered as an effective predictor of pancreatic related complications[7, 11, 12]. However, we did not realize to detect the amylase concentration in peritoneal drainage fluid until the patients had abnormal symptoms after the therapeutic prone position, which indicated that we lacked prediction in the diagnosis and treatment of the disease. Whether the patient had a biochemical leak prior to prone position or not was unknown.

Therefore, whether the amylase concentration in the drainage fluid on the first day after gastric cancer surgery can be used as a useful potential risk factor for pancreatic related complications needs further study.

\section{Conclusion}


The management of patients with large-scale abdominal surgery needs multidisciplinary cooperation. Surgeons should be gentle, maintain the intact pancreatic capsule as far as possible, and minimize pancreatic injury during operation. We should consider the advantages and disadvantages of HIPEC in patients with abdominal tumor resection, and pay more attention to the stimulation of temperature and chemotherapy drugs on abdominal organs. Critical physicians need to be fully aware of the details of the operation and should be circumspect to choose prone position ventilation strategy in the treatment of perioperative ARDS patients. If it is unavoidable to choose prone position ventilation in order to improve the body's hypoxia and oxygenation index, we can try to pad swimming circle around the abdomen and expose the position of abdominal median incision or internal organs to relieve abdominal visceral extrusion(Fig. 2). Meanwhile, we should closely monitor the changes of vital signs and the fluctuation of amylase in drainage fluid and serum.

\section{Abbreviations}

ARDS=acute respiratory distress syndrome, $C T=$ computed tomography, HIPEC= heated intraperitoneal chemotherapy, LRG= laparoscopic radical gastrectomy, OI= oxygenation index, PEEP=positive end expiratory pressure.

\section{Declarations}

\section{Acknowledgements}

Not applicable.

\section{Funding}

The author(s) received no financial support for the research, authorship, and/or publication of this article.

\section{Availability of data and materials}

Not applicable.

\section{Authors' contributions}

AX and WXW collected the data of this case, drafted the case report and were the major contributors in the writing and revising of the manuscript. LL performed the radiodiagnostical investigation of this case. DSK and WS contributed to the writing and drafting of the manuscript. LKL, HQS and ZLJ participated in the data collection and revision of the article. All authors read and approved the final manuscript.

\section{Ethics approval and consent to participate}

Not applicable.

Consent for publication 
Written informed consent was obtained from the patient for publication of this manuscript and any accompanying images. A copy of the written consent is available for review by the Editor-in-Chief of this journal.

\section{Competing interests}

The authors declare that they have no competing interests.

\section{Author details}

${ }^{1}$ Respiratory Department, Hospital of Chengdu University of Traditional Chinese Medicine, Chengdu, China.

${ }^{2}$ Clinical Medical College, Chengdu University of Traditional Chinese Medicine, Chengdu, China.

${ }^{3}$ Department of Critical Medicine, Hospital of Chengdu University of Traditional Chinese Medicine, Chengdu, China.

${ }^{4}$ Radiology department, Hospital of Chengdu University of Traditional Chinese Medicine, Chengdu, China.

\section{References}

1. Guérin C, Reignier J, Richard JC, et al.Prone positioning in severe acute respiratory distress syndrome. The New England journal of medicine.2013;368:2159-68.

2. Gaudry S, Tuffet $S$, Lukaszewicz AC, et al.Prone positioning in acute respiratory distress syndrome after abdominal surgery: a multicenter retrospective study : SAPRONADONF (Study of Ards and PRONe position After abDOmiNal surgery in France). Annals of intensive care.2017;7:21.

3. Bassi C, Marchegiani G, Dervenis C, et al.The 2016 update of the International Study Group (ISGPS) definition and grading of postoperative pancreatic fistula: 11 Years After. Surgery.2017;161:584-91.

4. Sano T, Sasako M, Yamamoto S, et al.Gastric cancer surgery: morbidity and mortality results from a prospective randomized controlled trial comparing D2 and extended para-aortic lymphadenectomyJapan Clinical Oncology Group study 9501. Journal of clinical oncology: official journal of the American Society of Clinical Oncology.2004;22:2767-73.

5. Yonemura Y, Wu CC, Fukushima N, et al. East Asia Surgical Oncology Group. Operative morbidity and mortality after D2 and D4 extended dissection for advanced gastric cancer: a prospective randomized trial conducted by Asian surgeons. Hepatogastroenterology. 2006;53:389-94.

6. Ichikawa D, Kurioka H, Yamaguchi T, et al. Postoperative complications following gastrectomy for gastric cancer during the last decade. Hepatogastroenterology. 2004;51:613-7.

7. Miki Y, Tokunaga M, Bando E, et al.Evaluation of postoperative pancreatic fistula after total gastrectomy with D2 lymphadenectomy by ISGPF classification. Journal of gastrointestinal surgery : official journal of the Society for Surgery of the Alimentary Tract.2011;15:1969-76. 
8. Hiki N, Honda M, Etoh $\mathrm{T}$, et al. Higher incidence of pancreatic fistula in laparoscopic gastrectomy. Real-world evidence from a nationwide prospective cohort study. Gastric Cancer. 2018;21:162-170.

9. Tsujiura M, Hiki N, Ohashi M, et al."Pancreas-Compressionless Gastrectomy": A Novel Laparoscopic Approach for Suprapancreatic Lymph Node Dissection. Annals of surgical oncology.2017;24:3331-7.

10. Zhaoqing T, Gang Z, Lu Z, et al. A multicenter prospective study on incidence and risk factors of postoperative pancreatic fistula after radical gastrectomy: a report of 2089 cases. Chinese Journal of Digestive Surgery.2020;19:63-71.

11. Iwata $\mathrm{N}$, Kodera $\mathrm{Y}$, Eguchi $\mathrm{T}$, et al.Amylase concentration of the drainage fluid as a risk factor for intra-abdominal abscess following gastrectomy for gastric cancer. World journal of surgery.2010;34:1534-9.

12. Seo KW, Yoon KY, Lee SH, et al.Amylase, lipase, and volume of drainage fluid in gastrectomy for the early detection of complications caused by pancreatic leakage. Journal of the Korean Surgical Society.2011;81:402-7.

\section{Figures}
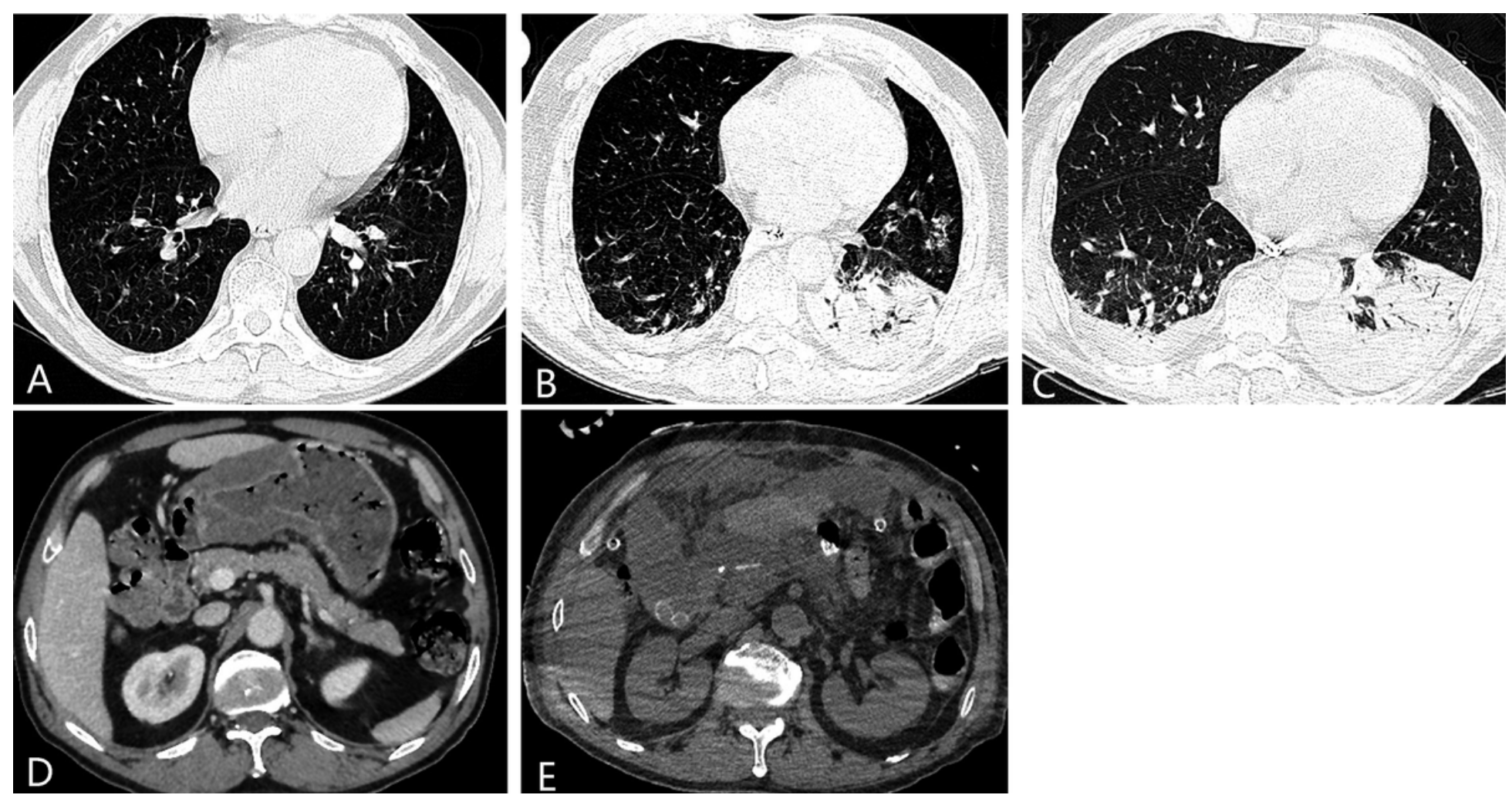

Figure 1

A: preoperative nonenhanced chest CT image. B: nonenhanced chest CT image obtained on the operative day. $C \bigotimes$ nonenhanced chest $C T$ image obtained 5 days after operation. D: preoperative nonenhanced abdominal CT image. E: nonenhanced abdominal CT image obtained 5 days after operation. 


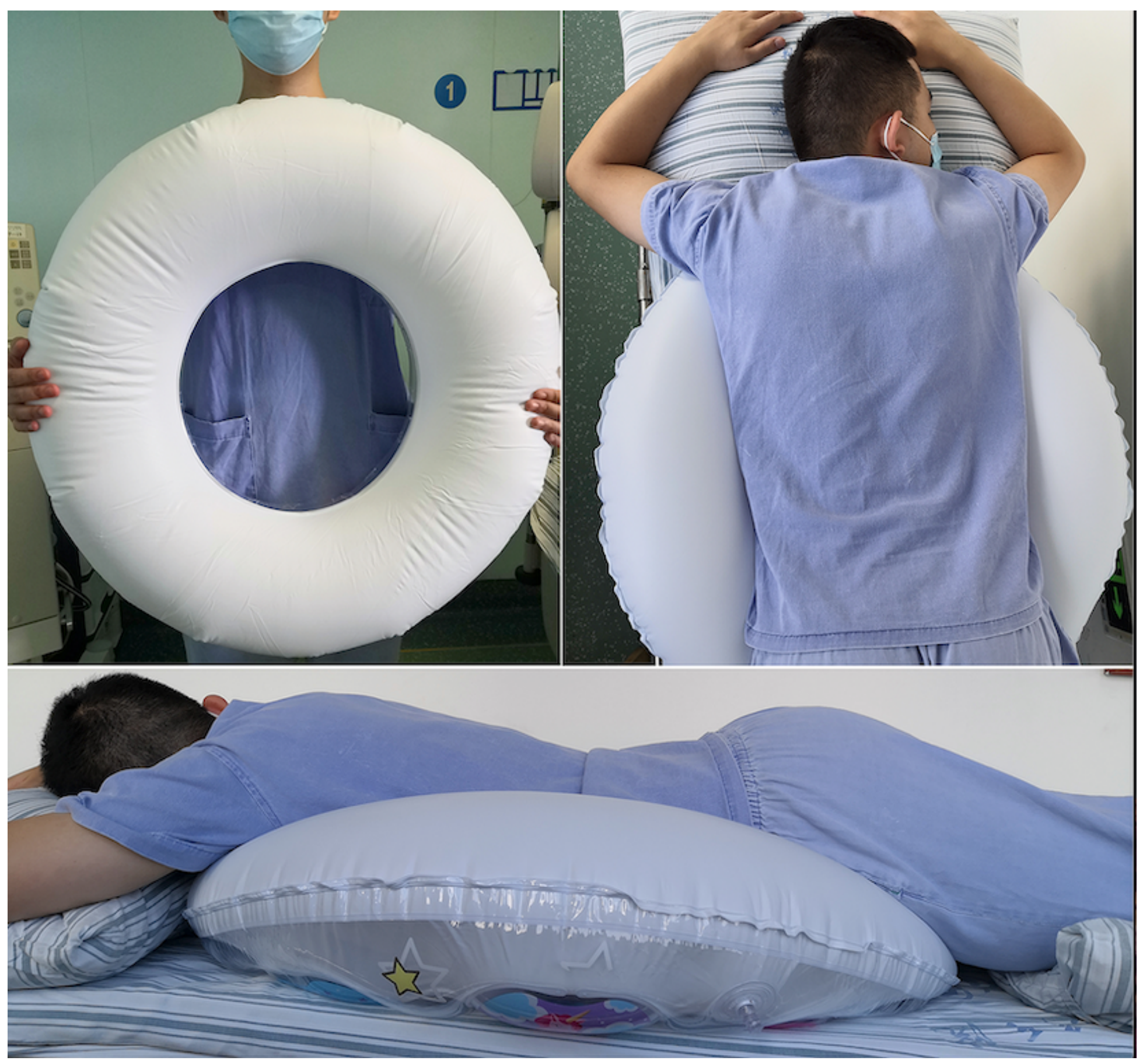

Figure 2

Placement of swimming ring in prone position 